\title{
Methods for Detection of Oxacillin Resistance among Coagulase-Negative Staphylococci Recovered from Patients with Bloodstream Infections at the University Hospital in Brazil
}

\author{
Deise Rafaela Ustulin and Maria de Lourdes Ribeiro de Souza da Cunha \\ Department of Microbiology and Immunology, Institute of Biosciences, UNESP - Univ. Estadual Paulista, \\ Botucatu, São Paulo, Brazil
}

\begin{abstract}
Coagulase-negative staphylococci (CoNS) are among the main causative agents of nosocomial infections, and multi-resistant strains has become a major issue over the past decades. The objectives of this study are to determine the frequency of oxacillin resistance among CoNS strains isolated from blood cultures of patients admitted at the University Hospital of the Faculty of Medicine of Botucatu, Brazil, and to compare phenotypic methods with the gold standard (amplification of the mecA gene) used for the detection of methicillin resistant-CoNS. Among the 103 CoNS strains analyzed, 85 were identified as $S$. epidermidis, ten as $S$. warneri, five as $S$. haemolyticus, one as $S$. capitis, one as $S$. hominis and one as $S$. lugdunensis. Amplification of the mecA gene revealed $85(82.5 \%)$ positive strains. Of these, $81(95.3 \%)$ were resistant by the oxacillin and cefoxitin disk diffusion method, 82 (96.5\%) by screening on Mueller-Hinton agar supplemented with $4 \mu \mathrm{g} / \mathrm{ml}$ oxacillin and $4 \% \mathrm{NaCl}$ and $84(98.8 \%)$ by the E-test. The mecA gene was detected in $82.3 \%$ of the $S$. epidermidis isolates and in seven of the ten $S$. warneri isolates. Among the phenotypic methods studied, the E-test yielded the best results when compared to the gold standard. The results showed that most of the CoNS strains were resistant to oxacillin and to multiple drugs; a fact making treatment of infections caused by these microorganisms more difficult.
\end{abstract}

Key words: Coagulase-negative Staphylococcus, Resistance, Oxacillin, mecA, E-test.

\section{Introduction}

Coagulase-negative staphylococci (CoNS) are reported to be the most common agent of nosocomial bloodstream infections (Wisplinghoff et al., 2004). The increasing characterization of infections caused by CoNS has proportionally increased the interest in the study of their susceptibility to different antimicrobial agents (Agvald-Öhman et al., 2004). Antibiotic resistance is easily acquired by these microorganisms; and the high rate of nosocomial transmission of plasmids between strains and the abusive use of antimicrobial drugs are important factors for the transfer of resistance genes and selection of multiresistant strains (McDonnell et al., 1983).

The prevalence of oxacillin-resistant CoNS has increased in most countries. Intrinsic resistance of Staphylococcus to oxacillin is mediated by the production of a supplemental penicillin-binding protein, called PBP 2a, which shows low affinity for semisynthetic penicillins. The mecA gene is

Copyright (C) 2012 Deise Rafaela Ustulin and Maria de Lourdes Ribeiro de Souza da Cunha. This is an open access article distributed under the Creative Commons Attribution License unported 3.0, which permits unrestricted use, distribution, and reproduction in any medium, provided that original work is properly cited. Contact author: Maria de Lourdes Ribeiro de Souza da Cunha E-mail: cunhamrl@ibb.unesp.br 
the genetic determinant of this protein and is located on the bacterial chromosome. This gene is identical in all staphylococcal strains and is therefore a useful marker of oxacillin resistance (Archer and Niemeyer, 1994). Although resistance mediated by the mecA gene is present in all cells of the population with intrinsic resistance, the gene may only be expressed by a low percentage of these cells, a fact resulting in the phenomenon of heterogenous resistance. This resistance can be classified into four classes of phenotypic expression (classes 1 to 4 ), where class 1 is the most heterogenous and class 4 the most homogenous (Tomasz et al., 1991).

Since phenotypic methods for the detection of methicillin-resistant Staphylococcus sometimes yield questionable results, molecular techniques for the detection of the mecA gene or its product PBP 2a have been proposed. Amplification of the mecA gene by the polymerase chain reaction (PCR) is the gold standard for the detection of methicillinresistant staphylococci (CLSI 2009). According to the Clinical and Laboratory Standards Institute (CLSI 2009), methods for the detection of the mecA gene, or of the protein encoded by this gene (PBP 2a), are the most adequate for the determination of oxacillin resistance and can be used to confirm the results of disk diffusion tests in the case of more severe infections. Other modalities of resistance have been described in strains that do not carry the mecA gene, which are called borderline resistance. There are two mechanisms of borderline resistance: the first is the inactivation of oxacillin mediated by the overproduction of $\beta$ lactamase, and the second is modified resistance (MOD-SA) which is mediated by intrinsic PBPs with altered affinity for oxacillin (McDougal and Thornsberry 1986; Tomasz et al., 1989).

The prevalence rates of methicillin-resistant coagulase-negative staphylococci (MRCoNS) show wide variations depending on the size and type of institution studied. Therefore, the main objective of the present study is to evaluate oxacillin susceptibility in CoNS strains isolated from blood cultures of patients admitted at the University Hospital of the Faculty of Medicine of Botucatu (HCFMB), Brazil, using different methods in order to optimize the isolation of MRCoNS.

\section{Materials and Methods}

\section{Bacterial Isolates}

The study was conducted in the Clinics Hospital of the Botucatu Faculty of Medicine of São Paulo State University, Brazil (HCFMB-UNESP). This is a teaching hospital with 400 active beds that serves an area of approximately one million inhabitants. A total of 103 CoNS strains isolated from blood cultures of patients hospitalized in different wards of HC-FMB between 2003 and 2007 and stored in the culture collection of the institution were studied. The strains were isolated as described by Koneman et al. (1997).

\section{Identification of Coagulase-Negative Staphylococci}

The isolates obtained from clinical specimens were seeded onto blood agar and stained by the Gram method for the evaluation of their purity and observation of morphology and specific staining. After confirmation of these characteristics, the isolates were submitted to catalase and coagulase tests. The genus Staphylococcus was differentiated from Micrococcus based on the oxidation and fermentation of glucose, resistance to bacitracin $(0.04 \mathrm{U})$ was indicated by the absence of an inhibition halo or the formation of a halo $<9 \mathrm{~mm}$ and susceptibility to furazolidone $(100 \mathrm{mg})$ indicated by inhibition halos measuring 15 to $35 \mathrm{~mm}$ in diameter (Koneman et al., 1997).

The coagulase test was used for the differentiation of $S$. aureus from CoNS (Koneman et al., 1997). Staphylococcal species were identified using the simplified method proposed by Cunha et al. (2004). The following international CoNS reference strains were used as controls: $S$. epidermidis (ATCC 12228), S. simulans (ATCC 27851), $S$. 
warneri (ATCC 10209) and S. xylosus (ATCC 29979). After species confirmation, the isolates were stored in nutrient broth containing glycerol at $-70^{\circ} \mathrm{C}$.

\section{Determination of Methicillin-Resistant Coagulase-Negative Staphylococci}

Susceptibility to oxacillin was tested by the agar diffusion method using disks impregnated with oxacillin (1 $\mu \mathrm{g})$ and cefoxitin $(30 \mu \mathrm{g})$ according to the criteria of the CLSI (2009) and by screening on MuellerHinton agar supplemented with $4 \mu \mathrm{g} / \mathrm{ml}$ oxacillin and 4\% $\mathrm{NaCl}$ (Ferreira et al., 2003).

\section{Determination of the Minimum Inhibitory} Concentration of Antibiotics by the E-Test

The in vitro susceptibility of the CoNS isolates against erythromycin, netilmicin, trimethoprim-sulfamethoxazole, oxacillin and vancomycin was tested. Susceptibility to the different drugs is expressed as follows: minimum inhibitory concentration (MIC50), defined as the minimum concentration of the drug necessary for $50 \%$ inhibition of the bacterial population tested; MIC90, defined as the minimum concentration necessary for $90 \%$ inhibition of the bacterial population; range of MIC and proportion of strains sensitive to each drug according to the definition of the CLSI (2009).

Detection of the Methicillin Resistance (Meca) Gene in Coagulase-Negative Staphylococci by PCR

Total nucleic acid was extracted from CoNS strains cultured on blood agar, individually inoculated into BHI, and incubated for $24 \mathrm{~h}$ at $37^{\circ} \mathrm{C}$. Nucleic acid was extracted with the Illustra kit (GE Healthcare) according to manufacturer instructions.

PCR was carried out in an appropriate thermocycler using the parameters described by Murakami et al. (1991). The efficiency of the amplification reactions was evaluated by electrophoresis on $2 \%$ agarose gel prepared in 1.0 X TBE buffer and stained with ethidium bromide.

\section{Evaluation of $\beta$-Lactamase Production}

The production of $ß$-lactamase was evaluated by the chromogenic cephalosporin disk assay using nitrocefin as substrate (cefinase, BBL) according to the manufacturer's instructions.

\section{Overproduction of $\beta$-Lactamase}

The mecA gene-negative strains that were resistant to oxacillin by the phenotypic methods were tested for overproduction of ß-lactamase. Disks impregnated with amoxicillin $(20 \mu \mathrm{g})$ and clavulanic acid (10 $\mu \mathrm{g}$ ) were used. The susceptibility breakpoint was the formation of an inhibition halo $\geq 20$ $\mathrm{mm}$ after $24 \mathrm{~h}$ of incubation at $35^{\circ} \mathrm{C}$ (Ghoshal et al., 2004).

\section{Statistical Analysis}

Sensitivity and specificity were used as measures for the comparison of the oxacillin and cefoxitin disk diffusion and screening methods with PCR, the gold standard for the detection of intrinsic oxacillin resistance (Sox 1966). Sensitivity was calculated based on the proportion of CoNS isolates that tested positive by PCR (presence of the mecA gene) and that were resistant to oxacillin by the phenotypic methods (oxacillin and cefoxitin disk diffusion, screening and E-test). Specificity was calculated as the proportion of CoNS isolates that tested negative by PCR (absence of the mecA gene) and that were sensitive to oxacillin by the phenotypic methods.

\section{Results}

\section{Identification of Coagulase-Negative Staphylococci}

Among the 103 isolates studied, 85 were identified as $S$. epidermidis, ten as $S$. warneri, five as $S$. haemolyticus, one as $S$. capitis, one as $S$. hominis, and one as $S$. lugdunensis. 


\section{Detection of the Methicillin Resistance in Coagulase-Negative Staphylococci}

The mecA gene was detected in 85 (82.5\%) of the 103 CoNS isolates studied (Table 1).

Table 1: Determination of Oxacillin Susceptibility in Coagulase-Negative Staphylococci by Phenotypic and Genotypic Methods

\begin{tabular}{|c|c|c|c|c|c|c|c|c|c|c|c|c|c|c|c|c|}
\hline \multirow[t]{5}{*}{ PCR $^{\mathbf{a}}$} & \multicolumn{16}{|c|}{ Phenotypic methods } \\
\hline & \multicolumn{8}{|c|}{ Disk diffusion } & & & & & & & & \\
\hline & \multicolumn{4}{|c|}{ Oxacillin } & \multicolumn{4}{|c|}{ Cefoxitin } & \multicolumn{4}{|c|}{ Screening } & \multicolumn{4}{|c|}{ E-test } \\
\hline & \multicolumn{2}{|c|}{$\mathbf{S}$} & \multicolumn{2}{|c|}{$\mathbf{R}$} & \multicolumn{2}{|c|}{$\mathbf{S}$} & \multicolumn{2}{|c|}{$\mathbf{R}$} & \multicolumn{2}{|c|}{$\mathbf{S}$} & \multicolumn{2}{|c|}{$\mathbf{R}$} & \multicolumn{2}{|c|}{$\mathbf{S}$} & \multicolumn{2}{|c|}{$\mathbf{R}$} \\
\hline & $\mathbf{N}$ & $\%$ & $\mathbf{N}$ & $\%$ & $\mathbf{N}$ & $\%$ & $\mathbf{N}$ & $\%$ & $\mathbf{N}$ & $\%$ & $\mathbf{N}$ & $\%$ & $\mathbf{N}$ & $\%$ & $\mathbf{N}$ & $\%$ \\
\hline $\begin{array}{l}\text { mecA+ } \\
(\mathrm{N}=85)\end{array}$ & 4 & 3.9 & 81 & 78.6 & 4 & 3.9 & 81 & 78.6 & 3 & 3.0 & 82 & 79.6 & 1 & 1.0 & 84 & 81.5 \\
\hline $\begin{array}{l}\text { mecA- } \\
(\mathrm{N}=18)\end{array}$ & 15 & 14.5 & 3 & 3.0 & 15 & 14.5 & 3 & 3.0 & 15 & 14.5 & 3 & 2.9 & 15 & 14.5 & 3 & 3.0 \\
\hline Total $(\mathrm{N}=103)$ & 19 & 18.4 & 84 & 81.6 & 19 & 18.4 & 84 & 81.6 & 18 & 17.5 & 85 & 82.5 & 16 & 15.5 & 87 & 84.7 \\
\hline Sensitivity (\%) & & & & & & & & & & & 5.5 & & & & & \\
\hline Specificity (\%) & & & & & & 83 & & & & & 3.3 & & & & & \\
\hline
\end{tabular}

Figure 1 shows the distribution of CoNS according to species and oxacillin resistance. Oxacillin resistance was detected by the gold standard method (amplification of the mecA gene) in $82.3 \%$ of $S$. epidermidis isolates and in seven of the ten $S$. warneri isolates. All five $S$. haemolyticus isolates were resistant to oxacillin, as well as the single isolate of $S$. capitis, S. hominis and S. lugdunensis.

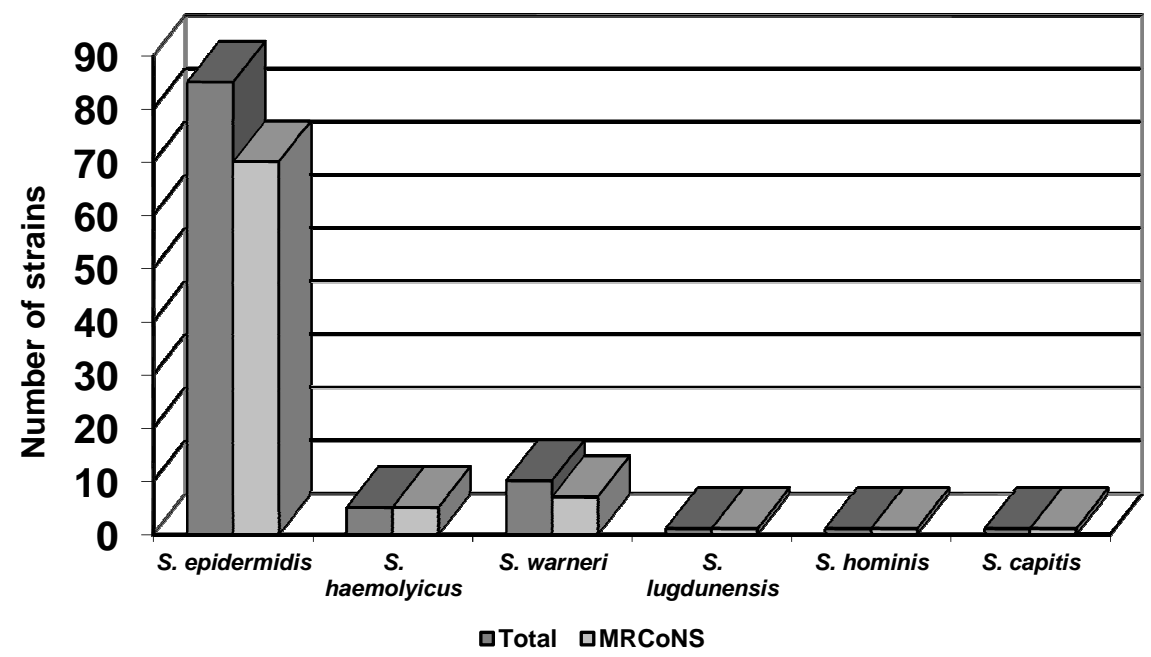

Fig. 1. Distribution of Coagulase-Negative Staphylococci according to Species and Oxacillin Resistance.

Eighty-four (81.6\%) of the 103 CoNS strains isolated from blood cultures of patients hospitalized in different wards of HC-FMB were resistant when tested by the oxacillin and cefoxitin disk diffusion methods, and eighty-five (82.5\%) were resistant to oxacillin by the screening test (Table 1 ).

For the 103 CoNS strains analyzed, the sensitivity of the oxacillin and cefoxitin disk diffusion methods was the same (95.3\%) and 
the sensitivity of the screening method was $96.5 \%$. A higher sensitivity was observed for the E-test (98.8\%). There was no difference in specificity between the methods tested $(83.3 \%)$.

\section{Determination of the Minimum Inhibitory Concentration of Antibiotics by the E-Test}

Eighty-seven (84.4\%) of the 103 CoNS strains studied were resistant to oxacillin by the E- test. Thirteen MRCoNS grew colonies within the E-test inhibition ellipse, a finding suggesting heterogenous resistance of these strains. The CoNS were also tested against other antibiotics. Seventy-one (68.9\%) were resistant to erythromycin, 57 (55.3\%) to netilmicin, $65(63.1 \%)$ to sulfamethoxazoletrimethoprim, and all were sensitive to vancomycin (Table 2).

Table 2: Antibiotic Resistance of Methicillin-Resistant and Sensitive Coagulase-Negative Staphylococci Detected by the E-Test

\begin{tabular}{|l|c|c|c|c|c|c|}
\hline \multirow{2}{*}{ Drug } & \multicolumn{2}{c|}{$\begin{array}{c}\text { MRCoNS } \\
\text { (N=85) }\end{array}$} & \multicolumn{2}{c|}{$\begin{array}{c}\text { MSCoNS } \\
\text { (N=18) }\end{array}$} & \multicolumn{2}{c|}{$\begin{array}{c}\text { Total } \\
\text { (N=103) }\end{array}$} \\
\cline { 2 - 7 } & $\mathbf{N}$ & $\mathbf{\%}$ & $\mathbf{N}$ & $\mathbf{\%}$ & $\mathbf{N}$ & \% \\
\hline Erythromycin* & 67 & 78.8 & 4 & 22.2 & 71 & 68.9 \\
\hline Netilmicin* & 54 & 63.5 & 3 & 16.7 & 57 & 55.3 \\
\hline Oxacillin* & 84 & 98.8 & 3 & 16.7 & 87 & 84.4 \\
\hline $\begin{array}{l}\text { Sulfamethoxazole- } \\
\text { trimethoprim* }\end{array}$ & 61 & 71.8 & 4 & 22.2 & 65 & 63.1 \\
\hline Vancomycin & 0 & 0 & 0 & 0 & 0 & 0 \\
\hline
\end{tabular}

*Intermediate results for these drugs were classified as resistant.

MRCoNS, coagulase-negative staphylococci resistant to oxacillin by PCR ( $m e c A+)$; MSCoNS, coagulase-negative staphylococci sensitive to oxacillin by PCR ( $\operatorname{mec} A-$-).

Among the CoNS strains tested against vancomycin, 19 (18.4\%) had MICs higher than $2 \mu \mathrm{g} / \mathrm{ml}$, including $18(17.5 \%)$ with an MIC of $3 \mu \mathrm{g} / \mathrm{ml}$ and one (0.9\%) with an MIC of $4 \mu \mathrm{g} / \mathrm{ml}$. Among the $S$. epidermidis isolates, there were 15 strains with vancomycin MICs of $3 \mu \mathrm{g} / \mathrm{ml}$ and one isolate with an MIC of 4 $\mu \mathrm{g} / \mathrm{ml}$. Two of the five $S$. haemolyticus isolates had MICs of $3 \mu \mathrm{g} / \mathrm{ml}$ and one $S$. warneri isolated presented an MIC of 3 $\mu \mathrm{g} / \mathrm{ml}$.

Susceptibility testing of the $S$. epidermidis strains to different drugs by the E-test showed a rate of oxacillin resistance of 98.6\%, sulfamethoxazole-trimethoprim resistance of $72.9 \%$, netilmicin resistance of $70 \%$ and erythromycin resistance of $85.7 \%$. These rates were $100 \%, 100 \%, 71.4 \%$ and $71.4 \%$ for $S$. warneri. The five $S$. haemolyticus isolates were resistant to oxacillin, 4 were resistant to sulfamethoxazole-trimethoprim,
2 to netilmicin and 3 to erythromycin. The only $S$. capitis strain included in the study was resistant to oxacillin, sulfamethoxazoletrimethoprim, and erythromycin, whereas the only $S$. hominis strain was resistant to all drugs analyzed, except for vancomycin. The only $S$. lugdunensis strain included in the study was resistant to oxacillin, sulfamethoxazole-trimethoprim, and erythromycin.

MICs ranged from $>32$ to $>256 \mu \mathrm{g} / \mathrm{ml}$ for most CoNS isolates analyzed. For erythromycin, MIC50 and MIC90 values were $>256 \mu \mathrm{g} / \mathrm{ml}$. The MIC50 and MIC90 values for netilmicin were 24.0 and $>256 \mu \mathrm{g} / \mathrm{ml}$, respectively. For oxacillin, MIC50 and MIC 90 values > $256 \mu \mathrm{g} / \mathrm{ml}$ were observed. The MIC50 and MIC90 values for sulfamethoxazole- trimethoprim (1/19) were $>32 \mu \mathrm{g} / \mathrm{ml}$, and for vancomycin 2.0 and 3.0 $\mu \mathrm{g} / \mathrm{ml}$, respectively (Table 3 ). 
Table 3: Minimum Inhibitory Concentrations of the Antibiotics against Methicillin-Resistant and Sensitive Coagulase-Negative Staphylococci

\begin{tabular}{|l|c|c|c|c|}
\hline \multirow{2}{*}{ Drug } & \multicolumn{2}{|c|}{ MIC50 $^{\mathbf{a}}(\boldsymbol{\mu g} / \mathbf{m l})$} & \multicolumn{2}{c|}{ MIC90 $^{\mathbf{b}}(\boldsymbol{\mu g} / \mathbf{m l})$} \\
\cline { 2 - 5 } & MRCoNS & MSCoNS & MRCoNS & MSCoNS \\
\hline Erythromycin & $\mathbf{( N = 8 5 )}$ & $\mathbf{( N = 1 8 )}$ & $\mathbf{( N = 8 5 )}$ & $\mathbf{( N = 1 8 )}$ \\
\hline Netilmicin & $>256$ & 0.25 & $>256$ & $>256$ \\
\hline Oxacillin & 32.0 & 0.38 & $>256$ & $>256$ \\
\hline $\begin{array}{l}\text { Sulfamethoxazole- } \\
\text { Trimethoprim (1/19) }\end{array}$ & $>256$ & 0.19 & $>256$ & $>256$ \\
\hline Vancomycin & $>32$ & 0.094 & $>32$ & $>32$ \\
\hline
\end{tabular}

aLowest concentration of the antibiotic able to inhibit the growth of $50 \%$ of the isolates.

bLowest concentration of the antibiotic able to inhibit the growth of $90 \%$ of the isolates.

MRCoNS, oxacillin-resistant coagulase-negative staphylococci; MSCoNS, oxacillin-sensitive coagulase-negative staphylococci.

Analysis of the distribution of resistance to these drugs among MRCoNS and methicillinsensitive CoNS (MSCoNS) has shown a difference between the two groups, with the observation of a higher rate of resistance to erythromycin, sulfamethoxazoletrimethoprim and netilmicin among MRCoNS isolates. The rate of erythromycin resistance was higher among MRCoNS, with 67 (78.8\%) resistant strains, as was the rate of sulfamethoxazole-trimethoprim resistance with $61(71.8 \%)$ resistant strains, and netilmicin resistance with 54 (63.5\%) resistant strains. MIC50 values were higher among MRCoNS strains for all drugs tested, except for vancomycin. However, the vancomycin MIC90 value was higher for MRCoNS strains $(3.0 \mu \mathrm{g} / \mathrm{ml})$.

Multidrug resistance was mainly observed for Staphylococcus resistant to oxacillin, with $43(50.6 \%)$ of the MRCoNS isolates being resistant to three of the four drugs tested. On the other hand, 12 (66.7\%) MSCoNS were sensitive to all drugs tested compared to 6 (7\%) MRCoNS.

\section{Determination of $\beta$-Lactamase Production}

Ninety-two (89.3\%) of the 103 CoNS strains studied were producers of $ß$-lactamase. Three CoNS isolates were negative for the mecA gene but produced ß-lactamase (Table 4).

Table 4: Coagulase-Negative Staphylococci Overproducing ß-Lactamase

\begin{tabular}{|c|c|c|c|c|c|c|c|}
\hline & & & \multicolumn{2}{|c|}{ Disk diffusion } & & & \\
\hline Strain & mecA & $\begin{array}{c}\text { Screenin } \\
\text { ga }^{\mathrm{a}}\end{array}$ & Oxacillin & Cefoxitin & $\begin{array}{c}\text { E-test }^{\mathrm{b}} \\
(\mu \mathrm{g} / \mathrm{ml})\end{array}$ & ACA $^{c}$ & ß-Lactamase \\
\hline H-2533/05 & - & + & $0 \mathrm{~mm}(\mathrm{R})$ & $0 \mathrm{~mm}(\mathrm{R})$ & $>256$ & $27(S)$ & positive \\
\hline H-793/06 & - & + & $0 \mathrm{~mm}(\mathrm{R})$ & $0 \mathrm{~mm}(\mathrm{R})$ & $>256$ & $24(S)$ & positive \\
\hline H-158/07 & - & + & $0 \mathrm{~mm}(\mathrm{R})$ & $17 \mathrm{~mm}(\mathrm{R})$ & $>256$ & $25(S)$ & positive \\
\hline
\end{tabular}

aMueller-Hinton agar containing $4 \mu \mathrm{g} / \mathrm{ml}$ oxacillin $+4 \% \mathrm{NaCl}$.

b Oxacillin by the E-test

cAmoxicillin + clavulanic acid

\section{Overproduction of $\beta$-Lactamase}

Three of the 103 CoNS strains studied were negative for the mecA gene and resistant to oxacillin by the phenotypic methods used.
However, these isolates were producers of $ß$ lactamase and were sensitive to amoxicillinclavulanic acid, thus confirming the presence of oxacillin resistance mediated by $\beta$ lactamase overproduction (Table 4). 


\section{Discussion}

Oxacillin resistance represents a major problem in the treatment of staphylococcal infections, especially because of the heterogenous expression of this resistance (Tveten et al., 2004). In the present study, oxacillin resistance was detected by PCR in $85(82.5 \%)$ of the CoNS isolates studied. Similar results have been reported by Perez and d'Azevedo (2008), who reported a rate of $78.4 \%$ of oxacillin-resistant CoNS strains using amplification of the mecA gene as the gold standard.

In addition to detection of the mecA gene, the reference methods recommended by the CLSI for the detection of oxacillin resistance in CoNS include the determination of MICs by agar and broth dilution methods, disk diffusion method, screening on MuellerHinton agar supplemented with $4 \mu \mathrm{g} / \mathrm{ml}$ oxacillin and $4 \% \mathrm{NaCl}$ and, more recently, the cefoxitin disk diffusion test (NCCLS 1999, CLSI 2009). The present results show a sensitivity of $95.3 \%$ of the oxacillin disk diffusion method for the detection of MRCoNS. A sensitivity of $100 \%$ has been reported by Secchi et al. (2008). Studies evaluating the performance of disk diffusion for the detection of MRSA and MRCoNS have shown that this method is less reliable in the case of heterogenous strains, in which only few cells carry the gene and are PBP 2a positive (Felten et al., 2002). The specificity of the oxacillin disk diffusion method was $83.3 \%$ in the present study. This percentage agrees with Ferreira et al. (2003) who found a specificity of $84 \%$ when investigating CoNS isolated from patients in different Brazilian hospitals over a period of 10 years. Ghoshal et al. (2004) reported a sensitivity and specificity of disk diffusion of $84 \%$ and $91 \%$, respectively.

Cefoxitin is considered to be an excellent inducer of mecA gene expression (McKinney et al., 2001). The present study demonstrates $95.3 \%$ sensitivity of and $83.3 \%$ specificity of the cefoxitin disk diffusion method for the detection of CoNS. These values are lower than the $100 \%$ sensitivity and $100 \%$ specificity reported by Secchi et al. (2008). One explanation for this difference in sensitivity and specificity might be related to the heterogeneity of the strains. Although few studies report the degree of heterogeneity of the isolates tested, it is assumed that resistant strains not detected by diffusion methods are more heterogenous.

Since the cut-off values for oxacillin MIC have been reduced, the use of $6 \mu \mathrm{g} / \mathrm{ml}$ is no longer recommended by the CLSI as a screening test for CoNS. However, several studies reported that this technique is sensitive and can be used as a confirmatory test for the results of disk diffusion (Hussain et al., 1998; Cavassini et al., 1999; Louie et al., 2001). Ferreira et al. (2003) found that the screening test with 4 $\mu \mathrm{g} / \mathrm{ml}$ oxacillin showed $100 \%$ sensitivity and $100 \%$ specificity versus $94.2 \%$ sensitivity and $100 \%$ specificity when a concentration of $6 \mu \mathrm{g} / \mathrm{ml}$ had been used, indicating the former as a screening method for CoNS. The sensitivity and specificity of screening on Mueller-Hinton agar supplemented with 4 $\mu \mathrm{g} / \mathrm{ml}$ oxacillin and $4 \% \mathrm{NaCl}$ used in the present study were $96.5 \%$ and $83.3 \%$, respectively. MRCoNS are detected without major difficulties when appropriate conditions including the supplementation of Mueller-Hinton agar with $\mathrm{NaCl}$ and adequate temperature and incubation times as recommended by the CLSI are used. However, the detection of heterogenous strains is more difficult, even when reference methods are employed (Swenson, 2002).

The E-test, an alternative to the microdilution assay used for the determination of MICs, detected $87 \quad(84.4 \%)$ oxacillin-resistant strains. The sensitivity of the test for the detection of oxacillin resistance was $98.8 \%$, a value higher than that obtained for the other phenotypic methods. Shorman et al. (2008) also detected oxacillin resistance in $86.2 \%$ of CoNS isolates by the E-test. Novak et al. (1993) used the E-test for the analysis of 127 MRSA and 100 MSSA strains previously identified by other phenotypic methods, with 
the test showing $100 \%$ sensitivity and specificity.

The lower specificity values obtained in the present study for all phenotypic methods $(83.3 \%)$ were due to the presence of falsepositive results when compared to the gold standard (detection of the mecA gene). However, the three isolates with falsepositive results were resistant to oxacillin in all phenotypic tests, were positive for $B$ lactamase and were sensitive to amoxicillinclavulanic acid, suggesting resistance mediated by ß-lactamase overproduction. Thus, when excluding these strains based on the fact that they presented another resistance mechanism, the specificity of all phenotypic tests was $100 \%$.

One important aspect of the pathogenesis of CoNS is the high rate of resistance of these microorganisms to antimicrobial agents. In the present study, $63.5 \%$ of MRCoNS were also resistant to netilmicin compared to $16.7 \%$ of MSCoNS. In addition, MRCoNS presented a higher rate of resistance to erythromycin and sulfamethoxazoletrimethoprim. These characteristics have a substantial impact on health care, adversely affecting patient morbidity and mortality (De Giusti et al., 1999). Many CoNS carry plasmids that can be transferred by conjugation between different species, or even between CoNS and $S$. aureus. This mechanism is an important route of transmission of antimicrobial resistance determinants, especially those conferring resistance to aminoglycosides and ß-lactam (Huebner and Goldmann, 1999).

Resistance of CoNS to oxacillin is generally accompanied by resistance to multiple antimicrobial agents. Most CoNS isolates presented MICs ranging from $>32$ to $>256$ $\mu \mathrm{g} / \mathrm{ml}$ for all drugs studied, a fact that leads to an increased use of glycopeptides for empirical treatment and even for prophylaxis. However, the selection of glycopeptide resistance and the potential transmission of this resistance between species indicate the need to restrict the use of these antimicrobial drugs (De Giusti et al.,
1999). These data emphasize the importance of antimicrobial susceptibility testing and the early identification of MRCoNS so that $ß$ lactam antibiotics can be used in the case of sensitive microorganisms in an attempt to reduce the unnecessary administration of glycopeptides (Hussain et al., 2002).

The rate of methicillin resistance among CoNS causing nosocomial infections, particularly $S$. epidermidis and $S$. haemolyticus, generally exceeds $80 \%$ (Grosserode and Wenzel, 1991). In the present study, all but one $S$. epidermidis isolate were resistant to oxacillin, as well as all five $S$. haemolyticus strains studied. The glycopeptide vancomycin is the antibiotic of choice for the treatment of CoNS that are multiresistant to commonly used antibiotics (Kloss and Bannerman, 1994). However, Veach et al. (1990) isolated vancomycinresistant $S$. haemolyticus from patients treated for a prolonged period of time with this antibiotic. The $S$. haemolyticus strains isolated from these patients showed reduced susceptibility to this drug when compared to strains isolated before antibiotic therapy. In the present study, 19 CoNS strains presented vancomycin MICs above the susceptibility breakpoint, including $16 \mathrm{~S}$. epidermidis isolates, two $S$. haemolyticus isolates and one S. warneri isolate. Palazzo et al. (2005) reported the isolation of four vancomycinresistant CoNS strains from healthy individuals (employees of a school and a hospital in São Paulo). Three of these isolates were mecA positive and presented high vancomycin MICs $(16,32$, and $>256 \mu \mathrm{g} / \mathrm{ml})$.

The rate of resistance to netilmicin, erythromycin and trimethoprimsulfamethoxazole among $S$. epidermidis, the species most frequently isolated in the present study, was higher than $70 \%$. This percentage is slightly higher than the resistance rate of $60 \%$ reported by Michelin et al. (2005). This high resistance to other antimicrobial agents can be explained by the high rate of oxacillin resistance observed among these isolates, since resistance to 
oxacillin indicates cross-resistance to other classes of antibiotics.

Although the sensitivity of phenotypic methods using cefoxitin disks has been reported to be $100 \%$, lower sensitivity values were obtained in the present study for the phenotypic tests, especially the disk diffusion method. These findings highlight the importance of molecular tests for the confirmation of oxacillin resistance in CoNS isolated from severe infections in order to guarantee correct treatment and to prevent the spreading of MRCoNS in the hospital environment. The present results show a higher prevalence of MRCoNS in the intensive care unit. Interestingly, Palos (2006) observed a higher prevalence of healthcare workers carrying MRSA in intensive care units, in agreement with the present study. The higher prevalence of MRCoNS in the intensive care unit observed in the present study might be related to the invasive procedures performed in this unit and the conditions of the patient when admitted to this ward.

Although detection of the mecA gene is important for the characterization of oxacillin resistance, other modalities of methicillin resistance have been described in strains that do not carry the mecA gene. These modalities are called borderline resistance, which can be the result of two mechanisms: the first is the inactivation of oxacillin mediated by the overproduction of ß-lactamase, and the second is modified resistance (MOD-SA) which is mediated by intrinsic PBPs with altered affinity for oxacillin (McDougal and Thornsberry, 1986; Tomasz et al., 1989). In the present study, ßlactamase was produced by $89.3 \%$ of the CoNS isolates, a percentage slightly higher than the $71.8 \%$ reported by Cunha and Lopes (2002).

The present results also show the presence of three CoNS strains that were resistant to oxacillin by the four phenotypic methods and negative for the mecA gene. However, these isolates were producers of $ß$-lactamase and were found to be sensitive in the disk diffusion test with amoxicillin-clavulanic acid, confirming that these strains overproduce $ß$-lactamase. Overproduction of ß-lactamase is one oxacillin resistance mechanism described for CoNS (McDougal and Thornsberry, 1986; Montanari et al., 1990).

Adequate accuracy in the detection of oxacillin resistance mediated by the mecA gene is important for the clinical laboratory. Although the reference methods detect most oxacillin-resistant strains, there are two situations that require additional steps for the confirmation of sensitivity or resistance. The first situation is the occurrence of extremely heterogenous strains that are classified as sensitive by the reference methods. The second situation is the occurrence of borderline resistance, which should be distinguished from mecA-mediated resistance since the clinical significance of the latter is much higher. Experimental animal studies and clinical data have shown the efficacy of $\beta$-lactam antibiotics for the treatment of infections caused by strains that do not carry the mecA gene and present low levels of resistance (borderline) (Massanari et al., 1988; Thauvin-Eliopoulos et al., 1990). However, infections caused by isolates carrying the mecA gene require treatment with vancomycin (Kolbert et al., 1995).

The present results confirm the importance of the detection of oxacillin resistance as a useful marker for the choice of antimicrobial therapy, with the observation of multiresistance in MRCoNS strains and higher susceptibility to other antimicrobial agents in MSCoNS strains. The use of the best techniques for the detection of oxacillin resistance, such as cefoxitin disk diffusion, screening test and E-test, as well as detection of the mecA gene and PBP 2a for laboratories that have access to more expensive techniques, may help prevent the abusive and unnecessary use of vancomycin in the case of MSCoNS and reserve this drug for severe infections caused by MRCoNS. The present results also show that most of the 
CoNS strains studied were resistant to oxacillin and to multiple drugs, a fact which makes treatment of infections caused by these microorganisms more difficult, in addition to the possible transfer of these genes to more virulent $S$. aureus strains.

\section{Acknowledgment}

Financial Support: Fundação de Amparo à Pesquisa do Estado de São Paulo (FAPESP) and Conselho Nacional de Desenvolvimento Científico e Tecnológico (CNPq).

\section{References}

Agvald-Öhman, C., Lund, B. \& Edlund, C. (2004). "Multiresistant Coagulase-Negative Staphylococci Disseminate Frequently between Intubated Patients in a Multidisciplinary Intensive Care Unit," Critical Care, 8 (1) 42-47.

Archer, G. L. \& Niemeyer, D. M. (1994). "Origin and Evolution of DNA Associated With Resistance to Methicillin in Staphylococci," Trends in Microbiology, 2 (10) 343-347.

Cavassini, M., Wenger, A., Jaton, K., Dominique. S. B. \& Bille, J. (1999). "Evaluation of MRSA-Screen, A Simple Anti-PBP 2a Slide Latex Agglutination Kit, For Rapid Detection Of Methicillin Resistance In Staphylococcus Aureus," Journal of Clinical Microbiology, 37 (5) 1591-1594.

Clinical and Laboratory Standards Institute (2009). 'Performance Standards for Antimicrobial Susceptibility Testing,' 19th Information Supplement. M100-S19. Clinical and Laboratory Standards Institute (CLSI), Wayne, PA, 2009.

Cunha, M. L. R. \& Lopes, C. A. M. (2002). "Estudo Da Produção De b-Lactamase E Sensibilidade Às Drogas Em Linhagens De Estafilococos Coagulase-Negativa Isolados De Recém-Nascidos," Jornal Brasileiro De Patologia E Medicina Laboratorial, 38 (4) 281-290.
Cunha M. L. R. S., Sinzato, Y. K. \& Silveira, L. V. A. (2004). "Comparison of Methods for the Identification of Coagulase-Negative Staphylococci," Memórias Do Instituto Oswaldo Cruz, 99 (8) 855-860.

De Giusti, M., Pacifico, L., Tufi, D., Panero, A., Boccia, A. \& Chiesa, C. (1999). "Phenotypic Detection of Nosocomial Meca-Positive Coagulase-Negative Staphylococci from Neonates," The Journal of Antimicrobial Chemotherapy, 44 (3) 351-358.

Felten, A., Grandry, B., Lagrange, P. H. \& Casin, I. (2002). "Evaluation of Three Techniques for Detection of Low-Level Methicillin-Resistant Staphylococcus Aureus (MRSA) a Disk Diffusion Method with Cefoxitin and Moxalactam, the Vitek 2 System, and the MRSA-Screen Latex Agglutination Test," Journal of Clinical Microbiology, 40 (8) 2766-2771.

Ferreira, R. B. R., Iorio, N. L. P., Malvar, K. L., Nunes, A. P. F., Fonseca, L. S., Bastos, C. C. R. \& Santos, K. R. N. (2003). "Coagulase-Negative Staphylococci: Comparison of Phenotypic and Genotypic Oxacillin Susceptibility Tests and Evaluation of the Agar Screening Test by Using Different Concentrations of Oxacillin," Journal of Clinical Microbiology, 41 (8) 36093614.

Ghoshal, U., Prasad, K. N., Singh, M., Tiwari, D. P. \& Ayyagari, A. (2004). "A Comparative Evaluation of Phenotypic and Molecular Methods for the Detection of Oxacillin Resistance in Coagulase-Negative Staphylococci," Journal of Infection and Chemotherapy, 10 (2) 86-89.

Grosserode, M. H. \& Wenzel, R. P. (1991). "The Continuing Importance of Staphylococci as Major Hospital Pathogens," Journal of Hospital Infection, 19 (Supp.B) 3-17.

Huebner, J. \& Goldmann, D. A. (1999). "Coagulase-Negative Staphylococci: Role as Pathogens," Annual Review of Medicine, 50 (1) 223-236. 
Hussain, Z., Stoakes, L., John, M. A., Garrow, S. \& Fitzgerald, V. (2002). "Detection of Methicillin Resistance in Primary Blood Culture Isolates of Coagulase-Negative Staphylococci by PCR, Slide Agglutination, Disk Diffusion, and a Commercial Method," Journal of Clinical Microbiology, 40 (6) 22512253.

Hussain, Z., Stoakes, L., Lannigan, R., Longo, S. \& Nancekivell, B. (1998). "Evaluation of Screening and Commercial Methods for Detection of Methicillin Resistance in Coagulase-Negative Staphylococci," Journal of Clinical Microbiology, 36 (1) 273-274.

Kloss, W. E. \& Bannerman, T. L. (1994). "Update on Clinical Significance of CoagulaseNegative Staphylococci," Clinical Microbiology Reviews, 7(1):117-140.

Kolbert, C. P., Connolly, J. E., Lee, M. J. \& Persing, D. H. (1995). "Detection of the Staphylococcal MecA Gene by Chemiluminescent DNA Hybridization," Journal of Clinical Microbiology, 33 (8) 21792182.

Koneman, E. W., Allen, S. D., Janda, W. M., Schreckenberger, P. C. \& Winn, Jr. W. C. (1997). 'Color Atlas and Textbook of Diagnostic Microbiology,' 5th Ed., Lippincott, Philadelphia, USA.

Louie, L., Majury, A., Goofellow, J., Louie, M. \& Simor, A. E. (2001). "Evaluation of a Latex Agglutination Test (MRSA-Screen) for Detection of Oxacillin Resistance in Coagulase-Negative Staphylococci," Journal of Clinical Microbiology, 39 (11) 4149-4151.

Massanari, R. M., Pfaller, M. A., Wakesfield, G. T., Hammons, L. A., Mcnut, L. A., Woolson, R. F. \& Helms, C. M. (1988). "Implications of Acquired Oxacillin Resistance in Management and Control of Staphylococcus Aureus Infection," The Journal of Infectious Diseases, 158 (4) 702-709.

McDonnell, R. W., Sweeney, H. M. \& Cohen, S. (1983). "Conjugational Transfer of Gentamicin Resistance Plasmids Intra- and
Interspecifically in Staphylococcus Aureus and Staphylococcus Epidermidis," Antimicrobial Agents Chemotherapy, 23 (1) 151-160.

McDougal, L. K. \& Thornsberry, C. (1986). "The Role of Beta-Lactamase in Staphylococcal Resistance to PenicillinaseResistant Penicillins and Cephalosporins," Journal of Clinical Microbiology, 23 (5) 832839.

Mckinney, T. K., Sharma, V. K., Craig, W. A. \& Archer, G. L. (2001). "Transcription of the Gene Mediating Methicillin Resistance in Staphylococcus Aureus (MecA) Is Corepressed But Not Coinduced by Cognate MecA and Beta-Lactamase Regulators," Journal of Bacteriology, 183 (23) 6862-6868.

Michelin, L., Lahude, M., Araujo, P. R., Givanaz, D. S. H., Muller, G., Delamare, A. P. L., Costa, S. O. P. and Echeverrigaray, S. (2005). "Pathogenicity Factors and Antimicrobial Resistance of Staphylococcus Epidermidis Associated with Nosocomial Infections Occurring in Intensive Care Unit," Brazilian Journal of Microbiology, 36 (1) 17-23.

Montanari, M. P., Tonin, E., Biavasco F. \& Varaldo P. E. (1990). "Further Characterization of Borderline MethicillinResistant Staphylococcus Aureus and Analysis of Penicillin-Binding Proteins," Antimicrobial Agents and Chemotherapy, 34 (5) 911-913.

Murakami, K., Minamide K., Wada K., Nakamura, E., Teraoka, H. \& Watanabe, S. (1991). "Identification of MethicillinResistant Strains of Staphylococci by Polymerase Chain Reaction," Journal of Clinical Microbiology, 29(10)2240-2244.

National Committee for Clinical Laboratory Standards. (1999). 'Performance Standards for Antimicrobial Susceptibility Testing,' NCCLS Approved Standard M100-S9. NCCLS, Wayne, PA, USA. 
Novak, S. M., Hindler, J. \& Bruckner, D. A. (1993). "Reliability of Two Novel Methods, Alamar and E Test, for Detection of Methicillin-Resistant Staphylococcus Aureus," Journal of Clinical Microbiology, 31 (11) 3056-3057.

Palazzo I. C. V., Araujo, M. L. C. \& Darini, A. L. C. (2005). "First Report of VancomycinResistant Staphylococci Isolated from Healthy Carriers in Brazil," Journal of Clinical Microbiology. 43 (1) 179-185.

Palos, M. A. P. (2006). 'Staphylococcus Aureus E Staphylococcus Aureus Meticilina Resistentes (MRSA) Em Profissionais De Saúde E As Interfaces Com As Infecções Nosocomiais,' Tese De Doutorado, Universidade De São Paulo, São Paulo, Brasil.

Perez, L. R. R. \& D'Azevedo, P. A. (2008). "Evaluation of the Accuracy of Various Phenotypic Tests to Detect Oxacillin Resistance in Coagulase-Negative Staphylococci," Brazilian Journal of Infectious Diseases, 12 (3) :210-212.

Secchi, C., Antunes A. L. S., Perez, L. R. R., Cantarelli, V. V. \& D'Azevedo, P. A. (2008). "Identification and Detection of Methicillin Resistance in Non-Epidermidis CoagulaseNegative Staphylococci," Brazilian Journal of Infectious Diseases, 12 (4) 316-320.

Shorman, M. A., Atoom, A. M., Abuharfeil, N. M. \& Almajali, A. M. (2008). "Identification of Methicillin Resistant Staphylococcus Aureus (MRSA) and Methicillin Resistant CoagulaseNegative Staphylococcus (CoNS) in Clinical Settings," American Journal of Infectious Diseases, 4 (2) 156-161.

Sox, H. C. (1968). "Probability Theory in the Use of Diagnostic Tests. An Introduction to Critical Study of Literature," Annals of Internal Medicine, 104 (1) 60-66.

Swenson, J. M. (2002). "New Tests for the Detection of Oxacillin-Resistant Staphylococcus Aureus," Clinical Microbiology Newsletter, 24 (21) 159-163.
Thauvin-Eliopoulos, C., Rice, L. B., Eliopoulos, G. M. \& Moellering, Jr, R. C. (1990). "Efficacy of Oxacillin and Ampicillin-Sulbactam Combination in Experimental Endocarditis Caused by b-Lactamase-Hyperproducing Staphylococcus Aureus," Antimicrobial Agents and Chemotherapy, 34 (5) 728-732.

Tomasz, A., Drugeon, H. B., Lencastre, H. M., Jabes D., Mcdougal, L. \& Bille J. (1989). "New Mechanism for Methicillin Resistance in Staphylococcus Aureus: Clinical Isolates That Lack the PBP 2a Gene and Contain Normal Penicillin-Binding Proteins with Modified Penicillin-Binding Capacity," Antimicrobial Agents and Chemotherapy, 33 (11) 18691874.

Tomasz, A., Nachman, S. \& Leaf, H. (1991). "Stable Classes of Phenotypic Expression in Methicillin-Resistant Clinical Isolates of Staphylococci," Antimicrobial Agents and Chemotherapy, 35 (1) 124-129.

Tveten, Y., Jenkins, A., Digranes, A., Melby, K. K., Allum, A. G. \& Kristiansen, B. E. (2004). "Comparison of PCR Detection of Meca with Agar Dilution and E-Test for Clinical Isolates of Coagulase Negative Staphylococci," Clinical Microbiology and Infection 10 (5) 462-465.

Veach, L. A., Pfaller, M., Barret M. \& Wenzel, R. (1990). "Vancomycin Resistance in Staphylococcus Haemolyticus Causing Colonization and Bloodstream Infection," Journal of Clinical Microbiology, 28 (9) 20642068.

Wisplinghoff, H., Bischoff T., Tallent, S. M., Seifert H., Wenzel, R. P. \& Edmond, M. B. (2004). "Nosocomial Bloodstream Infections in US Hospitals: Analysis of 24,179 Cases from a Prospective Nationwide Surveillance Study," Clinical Infectious Disease, 39 (3) 309314. 